\title{
Dependence of Transient and Residual Calcium Dynamics on Action-Potential Patterning during Neuropeptide Secretion
}

\author{
Martin Muschol ${ }^{1}$ and B. M. Salzberg ${ }^{1,2}$ \\ Departments of ${ }^{1}$ Neuroscience and ${ }^{2}$ Physiology, University of Pennsylvania School of Medicine, Philadelphia, \\ Pennsylvania 19104-6074
}

\begin{abstract}
Secretion of the neuropeptide arginine vasopressin (AVP) from the neurohypophysis is optimized by short phasic bursts of action potentials with a mean intraburst frequency around $10 \mathrm{~Hz}$. Several hypotheses, most prominently action-potential broadening and buildup of residual calcium, have been proposed to explain this frequency dependence of AVP release. However, how either of these mechanisms would optimize release at any given frequency remains an open question. We have addressed this issue by correlating the frequency-dependence of intraterminal calcium dynamics and AVP release during action-potential stimulation.

By monitoring the intraterminal calcium changes with lowaffinity indicator dyes and millisecond time resolution, the signal could be dissected into three separate components: rapid $\mathrm{Ca}^{2+}$ rises $\left(\Delta\left[\mathrm{Ca}^{2+}\right]_{\mathrm{tr}}\right)$ related to action-potential depolarization, $\mathrm{Ca}^{2+}$ extrusion and/or uptake, and a gradual increase in residual calcium $\left(\Delta\left[\mathrm{Ca}^{2+}\right]_{\text {res }}\right)$ throughout the stimulus train. Action-potential stimulation modulated all three components in a manner depen-
\end{abstract}

dent on both the stimulation frequency and number of stimuli. Overall, the cumulative $\Delta\left[\mathrm{Ca}^{2+}\right]_{\mathrm{tr}}$ amplitude initially increased with $f_{\text {Stim }}$ and then rapidly deteriorated, with a maximum around $f_{\text {Stim }} \leq 5 \mathrm{~Hz}$. Residual calcium levels, in contrast, increased monotonically with stimulation frequency.

Simultaneously with the calcium measurements we determined the amount of AVP release evoked by each stimulus train. Hormone release increased with $f_{\text {stim }}$ beyond the peak in $\Delta\left[\mathrm{Ca}^{2+}\right]_{\mathrm{tr}}$ amplitudes, reaching its maximum between 5 and 10 $\mathrm{Hz}$ before returning to its $1 \mathrm{~Hz}$ level. Thus, AVP release responds to the temporal patterning of stimulation, is sensitive to both $\Delta\left[\mathrm{Ca}^{2+}\right]_{\mathrm{tr}}$ and $\Delta\left[\mathrm{Ca}^{2+}\right]_{\text {res }}$, and is optimized at a frequency intermediate between the frequency-dependent maxima in $\Delta\left[\mathrm{Ca}^{2+}\right]_{\mathrm{tr}}$ and $\Delta\left[\mathrm{Ca}^{2+}\right]_{\text {res }}$.

Key words: calcium dynamics; excitation-secretion coupling; arginine vasopressin; exocytosis; neurohypophysis; action potential; residual calcium
Secretion of the peptide hormones arginine vasopressin (AVP) and oxytocin (OXT) from the mammalian posterior pituitary is differentially regulated by the pattern of action-potential activity that evokes release (Poulain and Wakerley, 1982; Cazalis et al., 1985; Gainer et al., 1986; Bicknell, 1988). AVP secretion is optimized by phasic bursts of action potentials, whereas optimal OXT release is associated with sustained high-frequency stimulation. Frequencydependent action-potential broadening is thought to be one of the mechanisms involved in this pattern-dependent regulation of secretion (Gainer et al., 1986; Bourque, 1990; Jackson et al., 1991). Buildup of residual calcium levels during bursts of action potentials is considered another contributing factor (Jackson et al., 1991; Stuenkel and Nordmann, 1993; Stuenkel, 1994). In addition, P/Qtype $\mathrm{Ca}^{2+}$-channels are expressed in AVP terminals, but not OXT terminals, and are believed to play a direct role in AVP secretion in the rat (Wang et al., 1997).

The purpose of our present study was twofold. First, we aimed to resolve the relative changes of $\Delta\left[\mathrm{Ca}^{2+}\right]_{i}$ within a given stimulation train, i.e., on a millisecond time scale. This time resolution would enable us to track relative changes in the near-membrane level of intraterminal $\mathrm{Ca}^{2+}$, as well as in the kinetics of the $\mathrm{Ca}^{2+}$ extrusion and/or uptake process, and to follow the amplitude and kinetics of residual calcium levels during trains of action potentials. Furthermore, for a meaningful comparison of stimulation-induced $\left[\mathrm{Ca}^{2+}\right]_{\mathrm{i}}$

Received April 19, 2000; revised June 14, 2000; accepted June 26, 2000.

This work was supported by United States Public Health Service Grant NS16824 We are grateful to Dr. E. L. Stuenkel for useful discussions and, in particular, for bringing the EIA assay for AVP measurements to our attention. We also acknowledge Dr. Meyer Jackson for helpful comments on this manuscript, Dr. S. Kraner for her help in implementing the EIA assay, and Dr. J. Lindstrom for making his cold room and plate reader available. We have also received valuable input and support from our colleague Dr. A. L. Obaid.

Correspondence should be addressed to Dr. Brian M. Salzberg, Department of Neuroscience, 234 Stemmler Hall, Philadelphia, PA 19104-6074. E-mail: bmsalzbe@mail.med.upenn.edu.

Copyright (C) 2000 Society for Neuroscience $0270-6474 / 00 / 206773-08 \$ 15.00 / 0$ changes, on the one hand, and the corresponding amount of hormone secretion, on the other hand, we wanted to measure both quantities simultaneously from the same preparation. To accomplish these goals, we used the low-affinity calcium indicator dye Mag-Fluo-4, which improved the temporal resolution of the $\Delta\left[\mathrm{Ca}^{2+}\right]_{\mathrm{i}}$ records from $\sim 1 \mathrm{sec}$ (Jackson et al., 1991; Stuenkel and Nordmann, 1993; Stuenkel, 1994) to $\leq 1 \mathrm{msec}$. Concurrently, we applied a quantitative enzyme immunoassay (EIA) to sample aliquots withdrawn from the bathing saline immediately after a given stimulation train to assess the amount of evoked hormone secretion.

The improved temporal resolution of the $\Delta\left[\mathrm{Ca}^{2+}\right]_{i}$ measurements permitted us to isolate different components of $\Delta\left[\mathrm{Ca}^{2+}\right]_{\mathrm{i}}$ and to determine their distinct patterns of modulation within a given train of action potentials. We demonstrate that these patterns vary with both the frequency of stimulation and the number of action potentials within the train. We also show that AVP release is shaped by the detailed and distinct dependence of transient and residual calcium on the temporal patterning of stimulation.

Portions of this work have been published previously in abstract form (Muschol et al., 1999; Muschol and Salzberg, 2000).

\section{MATERIALS AND METHODS}

Preparation and apparatus. Details of the preparation and apparatus have been described previously (Salzberg et al., 1983, 1985, 2000a; Gainer et al., 1986; Obaid et al., 1989). Briefly, 30- to 60-d-old CD-1 female mice (Jackson Laboratories, Bar Harbor, ME) were sacrificed using $\mathrm{CO}_{2}$ asphyxiation followed by decapitation. The neurohypophysis, together with the pars intermedia and a short segment of the infundibular stalk, was separated from the anterior pituitary. The preparation was mounted in an optical recording chamber and perfused with oxygenated $\left(95 \% \mathrm{O}_{2}, 5 \%\right.$ $\mathrm{CO}_{2}$ ) physiological saline containing (in mM): $\mathrm{NaCl} 155$, KCl 5.6, $\mathrm{CaCl}_{2}$ $2.2, \mathrm{MgCl}_{2} 1$, glucose $10, \mathrm{HEPES} 20, \mathrm{pH} 7.4$, at a rate of $300 \mu \mathrm{l} / \mathrm{min}$. Trains of action potentials were generated in the nerve terminals of the neurohypophysis by field depolarization of their axons at the level of the infundibular stalk with a pair of Teflon-insulated platinum (90\%)-iridium $(10 \%)$ wires. A Master-8 pulse generator (A.M.P.I., Jerusalem, Israel), driving two ISO-Flex stimulus isolators (A.M.P.I.), provided the bipolar 
pulses of $500 \mu \mathrm{sec}$ duration that were used for field depolarization. All experiments were performed at room temperature $\left(23 \pm 2^{\circ} \mathrm{C}\right)$. All chemicals used were from Sigma (St. Louis, MO) unless indicated otherwise.

Fluorescence measurements of calcium changes. The neurohypophysis was incubated for $90 \mathrm{~min}$ in a physiological saline containing $5 \mu \mathrm{M}$ of the AM form of the low-affinity $\left(K_{\mathrm{D} \mathrm{Ca}}=22 \mu \mathrm{M}\right)$ calcium indicator dye Mag-Fluo-4 (Molecular Probes, Eugene, OR), $0.9 \%$ dimethyl sulfoxide, and $0.1 \%$ Pluronic F-127 (BASF Wyandotte, Wyandotte, MI). All fluorescence measurements were performed on an upright UEM Microscope (Zeiss, Jena, Germany) using a Nikon $10 \times$ Fluor objective $(\mathrm{NA}=0.5)$. Light from a $300 \mathrm{~W}$ tungsten halogen lamp was passed through a $470 \pm 20 \mathrm{~nm}$ excitation filter (Zeiss). Dye fluorescence was monitored at $\lambda>515 \mathrm{~nm}$ (Schott Optical Glass Co., Duryea, PA) with a single large-area photodiode (PV-444, Perkin Elmer Optoelectronics, Vaudreuil, Canada). The photocurrent was converted into a voltage signal using a custom-built two-stage sample-and-hold amplifier (Cellular and Molecular Physiology Electronics Shop, Yale University School of Medicine, New Haven, CT). The sample-and-hold circuitry enabled us to measure and subtract the resting fluorescence, $F_{0}$, before second-stage amplification of the small, stimulation-induced fluorescence changes, $\Delta F$, superimposed on the fluorescence background $F_{0}$. The output from the second-stage amplifier was filtered at $1 \mathrm{kHz}$ with a LPF-8 low-pass eight-pole Bessel filter (Warner Instrument Corporation, Hamden, CT) and acquired at 16-bit resolution with an AT-MIO-16XE-50 data acquisition board (National Instruments, Austin, TX) running under the control of LabView Software (National Instruments). The fluorescence changes, $\Delta F$, were corrected for dye bleaching by subtracting a stimulation-free reference record collected immediately after each measurement. Total measurement duration for the fluorescence records varied from $45 \mathrm{sec}$ at $1 \mathrm{~Hz}$ down to $6 \mathrm{sec}$ for all stimulation frequencies above $10 \mathrm{~Hz}$. In a typical experiment, the tissue was allowed to rest for $15 \mathrm{~min}$ between stimulation trains. Trapping of calcium ionophores (A23187, ionomycin) in the outer layers of the neurohypophysis prevented us from using them to convert the $\Delta F / F_{0}$ values into absolute changes in $\left[\mathrm{Ca}^{2+}\right]$.

Enzyme immunoassay of AVP release. The amount of AVP released during a given stimulus train was determined using a solid-phase EIA Assay kit (Assay Designs, Ann Arbor, MI). To counteract the proteolytic activity in the tissue, the protease inhibitors phenylmethanesulfonyl fluoride (0.1 mM; Fluka Chemical Corp., Ronkonkoma, NY), leupeptin (2 $\mu \mathrm{g} / \mathrm{ml})$, and aprotinin $\left(5.2 \times 10^{-3} \mathrm{TIU} / \mathrm{ml}\right)$ were added to the physiological saline. Immediately before stimulation, superfusion flow through the recording chamber was halted. After a $5 \mathrm{~min}$ waiting period, $350 \mu \mathrm{l}$ of the perfusate surrounding the tissue was collected and kept at $4^{\circ} \mathrm{C}$. A 96-well EIA kit was used to analyze the AVP content in the samples. At the end of the second incubation period, sample absorbance at $\lambda=405 \mathrm{~nm}$ was determined with a Titertek Multiskan MCC/340 plate reader (Flow Laboratories, McLean, VA). Absorbance readings were converted into absolute AVP concentrations by interpolation from the absorbance of eight AVP calibration standards fitted to a four-parameter logistic equation [for details, see Chan (1987)].

Data analysis. Fluorescence data were processed using the IGOR data analysis software (Wavemetrics, Lake Oswego, OR). We used a sevenpoint binomial smoothing algorithm to reduce noise in the $\Delta F / F_{0}$ traces. This considerably improved the reliability of subsequent peak-detection procedures without affecting the time course or amplitude of the $\Delta F / F_{0}$ changes. Furthermore, the amplitudes of the first stimulation-induced peak in $\Delta F / F_{0}$ from all records within one experiment were matched to each other. As can be seen in Figure $1 B$, these amplitudes were already within $10 \%$ of one another before normalization. Values for $\Delta F / F_{0}$ before and at the peak immediately after each stimulus were extracted using a custom macro. These values were used to determine the amplitudes of the transient $\mathrm{Ca}^{2+}$ rise, $\Delta\left[\mathrm{Ca}^{2+}\right]_{\text {tr }}$, and the change in residual calcium, $\Delta\left[\mathrm{Ca}^{2+}\right]_{\text {res }}$.

Potential artifacts: relative fluorescence change, $\Delta F / F_{0}$, and intraterminal $\mathrm{Ca}^{2+}$-dynamics. We were concerned about excluding contributions to or distortions of $\Delta F / F_{0}$ from sources other than stimulation-induced calcium changes inside the nerve terminals. Dye signals from AM-loaded preparations might represent a superposition of $\Delta\left[\mathrm{Ca}^{2+}\right]_{\mathrm{i}}$ changes from several cellular components within the preparation. The neurohypophysis (pars nervosa) comprises millions of nerve terminals and secretory swellings (Herring bodies), and these are the most abundant structure in this tissue, accounting for some $99 \%$ of the excitable membrane in rat (Nordmann, 1977). The only other cellular structures with significant total volume are pituicytes. Because pituicytes apparently lack voltage-gated calcium channels, we do not expect these glial elements to contribute to stimulationinduced changes of $\Delta F / F_{0}$. Furthermore, the considerably larger volume of pituicytes $\left(275 \mu \mathrm{m}^{3}\right)$ versus nerve endings $\left(2-14 \mu \mathrm{m}^{3}\right)$ (Nordmann, 1977), and their correspondingly smaller surface to volume ratio, should result in substantially higher dye concentrations inside AM-loaded nerve terminals than inside pituicytes. That expectation was confirmed by the dramatically lower resting fluorescence observed after AM loading of the large cells in the neighboring pars intermedia ( $>20 \mu \mathrm{M}$ diameter) (Bourque, 1990). This difference in dye concentration will significantly enhance the contributions of nerve terminals to $\Delta F / F_{0}$ signals. Another possible concern is the heterogeneity of the nerve terminal population itself, which contains both AVP- and OXT-secreting terminals. Although different types and distributions of $\mathrm{Ca}$ channels have been identified pharmacologically (Wang et al., 1997), patch-clamp measurements showed nerve terminals to be ho- mogeneous in their Ca-channel properties (Branchaw et al., 1997). Calcium measurements from isolated nerve terminals (Stuenkel, 1994; Fisher and Fernandez, 1999) or in tissue slices (Jackson et al., 1991) showed calcium kinetics to be insensitive to terminal peptide identity.

Uptake of AM-loaded dyes into subcellular compartments presents another potential source of $\Delta F / F_{0}$ distortions (Almers and Neher, 1985). Indeed, loading of Fura- 2 into the subcellular compartments of the rat neurohypophysis has been reported (Troadec et al., 1998). If compartmentalization occurs with Mag-Fluo-4, it does not appear to contribute to the stimulation-induced fluorescence changes. In Figure $1 B$ we have superimposed $\Delta F / F_{0}$ data from the first action potential at each stimulation frequency. Figure $1 C$ illustrates the corresponding values of the resting fluorescence $F_{0}$ for each of these measurements. Note that the $\Delta F / F_{0}$ amplitudes remain constant throughout the experiment, whereas loss of dye from the tissue typically resulted in a reduction in $F_{0}$ by a factor of 2 or more. Given this dye loss, the relative proportion of cytosolic versus compartmentalized dye is bound to change over time. If compartmentalized dye contributed to $\Delta F / F_{0}$, this dye redistribution in turn would alter $\Delta F / F_{0}$ amplitudes. This was not observed.

Dye properties such as binding kinetics, dye saturation, or sensitivity to $\mathrm{Mg}^{2+}$ can distort both the time course and the relative amplitudes of $\Delta F / F_{0}$ measurements (Baylor and Hollingworth, 1988; Helmchen et al., 1997). Mag-Fluo-4 is a low-affinity calcium indicator $\left(K_{\mathrm{D}, \mathrm{Ca}}=22 \mu \mathrm{M}\right)$ (Haugland, $1996)$ with fast on/off rates. Because we estimate the maximal dye concentration in the terminals at $100-200 \mu \mathrm{M}$, distortions of the time course of $\Delta F / F_{0}$ are not expected. In fact, the intrinsically fast kinetics of this indicator dye compared with its high-affinity variants (i.e., Fluo-4 or Fluo-3) enabled us to detect the rapidly changing components of $\Delta\left[\mathrm{Ca}^{2+}\right]_{\mathrm{i}}$. Distortions of $\Delta F / F_{0}$ amplitudes resulting from dye saturation are also negligible. For typical resting levels of intraterminal $\left[\mathrm{Ca}^{2+}\right]_{\mathrm{i}}$ of $100-300$ nM (Jackson et al., 1991; Stuenkel, 1994), the resting fluorescence $F_{0}$ of Mag-Fluo-4 will be close to its zero-calcium value, $F_{\text {free, }}$, whereas the dynamic range, $\left(F_{\text {bound }}-F_{\text {free }}\right) / F_{\text {free, }}$ of Mag-Fluo-4 exceeds a factor of 25 in our microscope measurement system. The maximal value of $\Delta F / F_{0}$ during measurements did not exceed $15 \%$, well within the compass of linear dye response. In addition, $\Delta F / F_{0}$ amplitudes obtained with $\mathrm{KCl}$ depolarization ranged from 50 to $200 \%$ without showing signs of dye saturation (data not shown).

Finally, to evaluate potential $\Delta \mathrm{Mg}^{2+}$ contributions to the measurements, we repeated $\Delta F / F_{0}$ measurement with two additional low-affinity $\mathrm{Ca}^{2+}$ indicators, Magnesium Green and Mag-Fura-2. The relative $\mathrm{Ca} / \mathrm{Mg}$ sensitivities among these three dyes differ by as much as a factor of 4 , with Mag-Fluo-4 being the least $\mathrm{Mg}^{2+}$ sensitive. Nevertheless, all three dyes yielded identical $\Delta F / F_{0}$ traces (results not shown). We therefore presume that $\mathrm{Mg}^{2+}$ changes make no discernable contribution to $\Delta F / F_{0}$ on the time scale of our measurements, in agreement with observations on nerve terminals in the Calyx of Held (Helmchen et al., 1997). On the basis of all of these arguments, we conclude that the observed fluorescence changes, $\Delta F / F_{0}$, reported below are linear and that they provide a reliable, if uncalibrated, representation of intraterminal calcium changes.

\section{RESULTS}

\section{Intraterminal calcium changes $\left(\Delta[\mathrm{Ca}]_{i}\right)$ during trains of action potentials}

Figure 1 presents a summary of intraterminal $\mathrm{Ca}^{2+}$ changes, $\Delta\left[\mathrm{Ca}^{2+}\right]_{\mathrm{i}}$, in the neurohypophysis resulting from stimulation with trains of action potentials $\left(N_{\text {Stim }}=40\right)$ at the indicated frequencies. For any given train, $\Delta\left[\mathrm{Ca}^{2+}\right]_{i}$ can be separated into three distinct phases. Each action potential triggers a rapid ( $\cong 15 \mathrm{msec})$, transient calcium rise, $\Delta\left[\mathrm{Ca}^{2+}\right]_{\mathrm{tr}}$. Between stimuli, a slower decay phase, $\Delta\left[\mathrm{Ca}^{2+}\right]_{\mathrm{dec}}$, related to $\mathrm{Ca}^{2+}$ extrusion and/or uptake, prevails. In addition, residual calcium, $\left[\mathrm{Ca}^{2+}\right]_{\text {res }}$, accumulates as a result of incomplete $\mathrm{Ca}^{2+}$ removal during the interstimulus period.

It is instructive to compare the overall shape of the observed $\Delta\left[\mathrm{Ca}^{2+}\right]_{\mathrm{i}}$ traces with the predictions from a simple onecompartment model of $\mathrm{Ca}^{2+}$ kinetics during repetitive stimulation (Regehr et al., 1994). This model accounts for periodic $\mathrm{Ca}^{2+}$ influx during stimulation trains, $\mathrm{Ca}^{2+}$ buffering by endogenous buffers and $\mathrm{Ca}^{2+}$ indicators, and a $\mathrm{Ca}^{2+}$ extrusion process linearly proportional to $\left[\mathrm{Ca}^{2+}\right]_{\mathrm{i}}$, the free calcium concentration in the terminal. For a typical nerve terminal of radius $r \cong 1-3 \mu \mathrm{m}$ (Nordmann, 1977; Sattelle, 1988), and presuming an intraterminal diffusion coefficient of $6 \times 10^{-6} \mathrm{~cm}^{2} / \mathrm{sec}$ (Blaustein and Hodgkin, 1969), the time for spatial $\mathrm{Ca}^{2+}$ gradients to dissipate can be estimated as $\tau_{\text {Diff }} \cong r^{2} / 6 \mathrm{D}_{\mathrm{Ca}} \leq 2.5 \mathrm{msec}$ (Crank, 1975). The neglect of diff usion effects is therefore a good approximation for the experimental situation with which we are concerned. The model readily reproduces the overall shape of the $\Delta\left[\mathrm{Ca}^{2+}\right]_{\mathrm{i}}$ traces. In particular, it predicts that for any given stimulation frequency, $f_{\text {Stim }}$, residual 

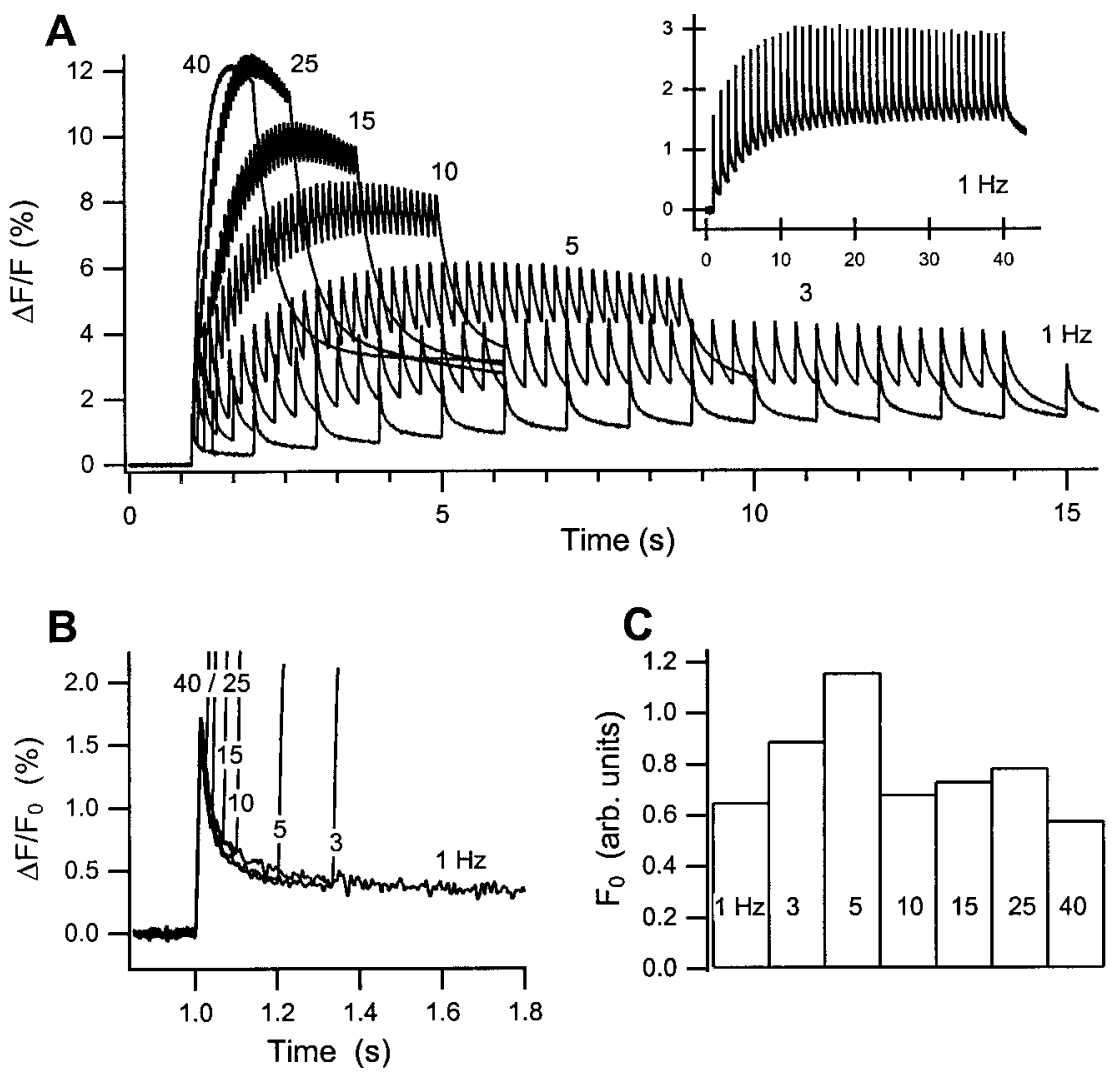

calcium levels will increase until they reach a steady-state plateau at which the $\mathrm{Ca}^{2+}$-dependent extrusion/uptake rate between stimuli exactly balances the $\mathrm{Ca}^{2+}$ rise during stimulation. The amplitude of the residual calcium plateau is expected to increase linearly with $f_{\text {Stim }}$, because the time-averaged $\mathrm{Ca}^{2+}$ influx is presumed constant. The model therefore provides an important reference point against which to compare the experimentally observed $\mathrm{Ca}^{2+}$ dynamics. In the remainder of this section we will explore the effects of various stimulation parameters on the transient $\mathrm{Ca}^{2+}$ rises $\left(\Delta\left[\mathrm{Ca}^{2+}\right]_{\mathrm{tr}}\right)$, $\mathrm{Ca}^{2+}$ extrusion/uptake $\left(\Delta\left[\mathrm{Ca}^{2+}\right]_{\mathrm{dec}}\right)$, and residual calcium changes $\left(\Delta\left[\mathrm{Ca}^{2+}\right]_{\mathrm{res}}\right)$, as well as their impact on AVP release.

\section{Facilitation and depression of $\Delta\left[\mathrm{Ca}^{2+}\right]_{\mathrm{tr}}$ amplitudes during trains of action potentials}

In Figure 2 we have plotted $\Delta\left[\mathrm{Ca}^{2+}\right]_{\operatorname{tr}}$ amplitudes versus the stimulus number, $n_{\text {Stim }}$, from the data in Figure 1 . Both stimulation frequency, $f_{\text {Stim }}$, and total number of stimuli, $N_{\text {Stim }}$, affect $\Delta\left[\mathrm{Ca}^{2+}\right]_{\text {tr }}$ amplitudes. At $f_{\text {Stim }}=1 \mathrm{~Hz}$, a barely perceptible facilitation of $\Delta\left[\mathrm{Ca}^{2+}\right]_{\mathrm{tr}}$ amplitudes occurs within the first few stimuli. As stimulation extends beyond 10 stimuli, $\Delta\left[\mathrm{Ca}^{2+}\right]_{\text {tr }}$ amplitudes begin to deteriorate, declining to $80 \%$ of their initial value at the end of a 40 stimulus train. At $f_{\text {Stim }}=3 \mathrm{~Hz}$, facilitation of early $\Delta[\mathrm{Ca}]_{\mathrm{tr}}$ amplitudes is dramatic, with the amplitude of the fifth response $>60 \%$ greater then the initial response. Again, as stimulation continues, $\Delta\left[\mathrm{Ca}^{2+}\right]_{\mathrm{tr}}$ deteriorates within the train. With increasing stimulation frequency, facilitation does not persist beyond the first few stimuli: e.g., at $15 \mathrm{~Hz}, \Delta\left[\mathrm{Ca}^{2+}\right]_{\text {tr }}$ returns to its initial amplitude within $<10$ stimuli. Above $15 \mathrm{~Hz}$, depression of $\Delta\left[\mathrm{Ca}^{2+}\right]_{\mathrm{tr}}$ dominates throughout the stimulation train. $\Delta\left[\mathrm{Ca}^{2+}\right]_{\mathrm{tr}}$ depression progresses further with $f_{\text {Stim }}$, until at $40 \mathrm{~Hz}$ the $\Delta\left[\mathrm{Ca}^{2+}\right]_{\text {tr }}$ amplitude at the end of 40 stimuli has deteriorated to only $20 \%$ of its value at the onset of the stimulus train.

Action-potential stimulation therefore produces three distinct patterns of $\mathrm{Ca}^{2+}$ rises as a function of frequency. At or below $f_{\text {Stim }}$ $=1 \mathrm{~Hz}, \Delta\left[\mathrm{Ca}^{2+}\right]_{\mathrm{tr}}$ amplitudes are nearly constant, with mild depression developing slowly during extended stimulation. Between 1 and $10 \mathrm{~Hz}$, early facilitation of $\Delta\left[\mathrm{Ca}^{2+}\right]_{\operatorname{tr}}$ amplitudes dominates throughout the stimulation train. Beginning at $\sim 10 \mathrm{~Hz}$,
Figure 1. Frequency dependence of intraterminal calcium changes. $A$, Superposition of the fluorescence changes, $\Delta F / F_{0}$, recorded with the low-affinity calcium indicator dye Mag-Fluo-4/AM $\left(K_{\mathrm{D}, \mathrm{Ca}}=22 \mu \mathrm{M}\right)$ in the mouse neurohypophysis during trains of 40 action potentials at the indicated frequencies. The fluorescence data were low-pass-filtered at $1 \mathrm{kHz}$ and corrected for dye bleaching by subtraction of a reference trace without stimulation. The data in $A$ were also passed through a sevenpoint smoothing algorithm, and their final amplitudes were matched to the amplitude of the first $\Delta\left[\mathrm{Ca}^{2+}\right]_{\mathrm{tr}}$ of the control trace at $15 \mathrm{~Hz}$. In the inset, the complete time course of the truncated $1 \mathrm{~Hz}$ fluorescence trace is shown. $B$, Initial $\Delta\left[\mathrm{Ca}^{2+}\right]_{\mathrm{tr}}$ amplitudes from the data in Figure 1 before matching their peaks. Even without peak matching, $\Delta F / F_{0}$ values are within $10 \%$ of one another. In contrast, the corresponding values for the resting fluorescence $F_{0}$ (not in chronological order) changed nearly twofold during the course of these recordings, as shown in $C$.

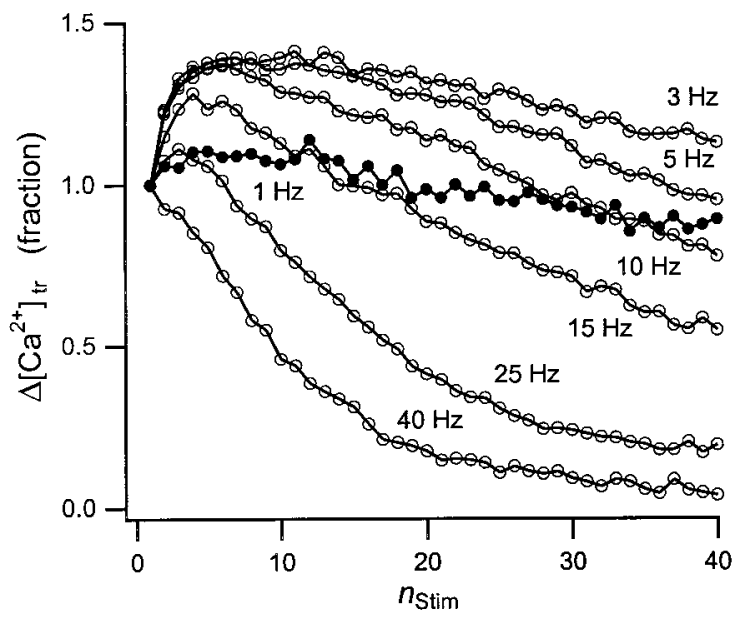

Figure 2. Amplitude variation of the transient $\mathrm{Ca}^{2+}$ rise. Shown are fractional changes in the amplitude of the transient $\mathrm{Ca}^{2+}$ rise, $\Delta\left[\mathrm{Ca}^{2+}\right]_{\mathrm{tr}}$, during a train of 40 action potentials at the indicated stimulation frequencies $(\mathbf{0}, 1 \mathrm{~Hz} ; \bigcirc$, all other frequencies), normalized to the first transient of each train. Three different patterns of facilitation and depression emerge. At low frequencies $\left(f_{\text {Stim }} \leq 1 \mathrm{~Hz}\right), \Delta\left[\mathrm{Ca}^{2+}\right]_{\text {tr }}$ amplitudes are essentially constant throughout the train $(\mathbf{0})$. At moderate frequencies $(1 \mathrm{~Hz}<$ $\left.f_{\text {Stim }} \leq 15 \mathrm{~Hz}\right), \Delta\left[\mathrm{Ca}^{2+}\right]_{\text {tr }}$ amplitudes facilitate during the initial stimuli but deteriorate later in the train. Finally, at higher frequencies, depression of $\Delta\left[\mathrm{Ca}^{2+}\right]_{\mathrm{tr}}$ amplitudes dominates throughout the train, resulting in $\Delta\left[\mathrm{Ca}^{2+}\right]_{\text {tr }}$ amplitudes deteriorating to $<20 \%$ of their initial values at the end of 40 stimuli.

early facilitation is eroded by subsequent depression. Overall, depression develops at progressively smaller $n_{\text {Stim }}$ and at an increasingly rapid rate as $f_{\text {Stim }}$ increases. Eventually, $\Delta\left[\mathrm{Ca}^{2+}\right]_{\text {tr }}$ amplitudes deteriorate monotonically throughout the train.

To evaluate the physiological significance of the observed facilitation and depression of $\Delta\left[\mathrm{Ca}^{2+}\right]_{\mathrm{tr}}$, we need to consider the factors that might contribute to $\Delta\left[\mathrm{Ca}^{2+}\right]_{\mathrm{tr}}: \mathrm{Ca}^{2+}$ influx, $\mathrm{Ca}^{2+}$ mobilization, and $\mathrm{Ca}^{2+}$ extrusion and uptake, as well as $\mathrm{Ca}^{2+}$ buffering by 

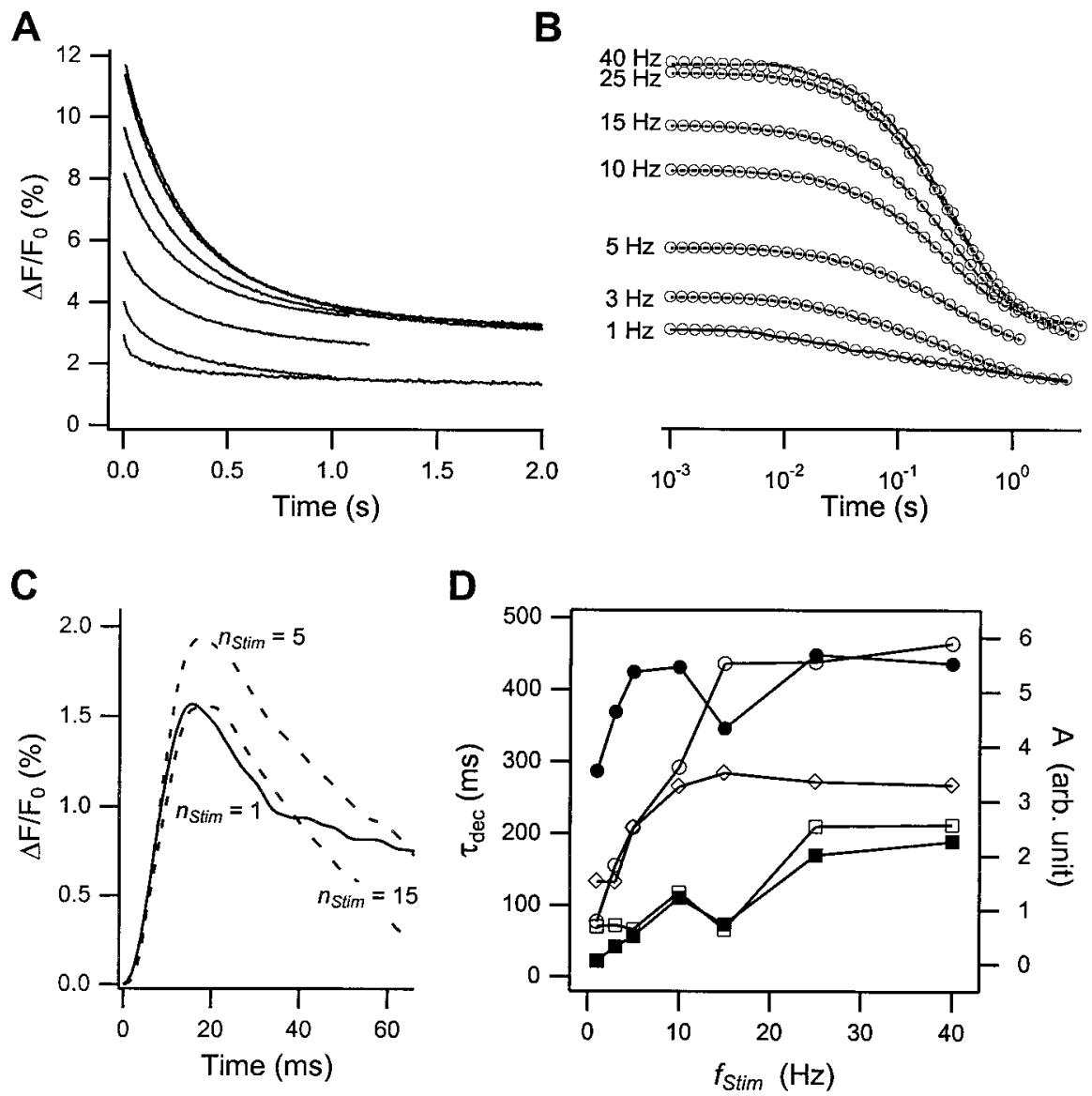

Figure 3. Decay of intraterminal calcium levels after stimulation. Shown is the decay of $\left[\mathrm{Ca}^{2+}\right]_{i}$ at the end of stimulation with 40 action potentials at the indicated $f_{\text {Stim }}$ on linear $(A)$ and logarithmic $(B)$ time scales. The $y$-axis scale in $B$ is identical to the one in $A$. The solid lines in $A$ represent the raw data points. In $B$ the raw data from $A$ were resampled uniformly on a logarithmic time scale. The open circles in $B$ are the results from double-exponential fits through the data. $C$, Changes in the shape of the decay phase of the 1 st $(-), 5$ th, and 15 th $(---) \Delta\left[\mathrm{Ca}^{2+}\right]_{\mathrm{i}}$ response during action-potential stimulation at $f_{\mathrm{Stim}}=$ $15 \mathrm{~Hz}$. D. Dependence of the amplitudes (open symbols) and time constants (closed symbols) of the double-exponential fits in $B$ on stimulation frequency ( $\diamond$, constant background; $\square$, $\mathbf{\square}$, first and $\bigcirc, \boldsymbol{\Theta}$, second fast component).

the endogenous $\mathrm{Ca}^{2+}$ buffers and the $\mathrm{Ca}^{2+}$ indicator dye. As we have argued in Materials and Methods, distortion of the intrinsic $\mathrm{Ca}^{2+}$ dynamics resulting from dye saturation, slow dye kinetics, or competition with the intrinsic $\mathrm{Ca}^{2+}$ buffers is negligible. Furthermore, $\mathrm{Ca}^{2+}$ binding by both dye and buffer and diffusion of $\mathrm{Ca}^{2+}$ in these small terminals are fast enough that the $\mathrm{Ca}^{2+}$-indicator dye is always in quasi-equilibrium with $\left[\mathrm{Ca}^{2+}\right]_{\mathrm{i}}$. Studies on isolated nerve terminals have shown that $>99 \%$ of the $\mathrm{Ca}^{2+}$ influx is rapidly bound to endogenous $\mathrm{Ca}^{2+}$ buffer(s), thereby dramatically limiting the amplitude of $\Delta\left[\mathrm{Ca}^{2+}\right]_{\text {tr }}$ (Stuenkel, 1994). Saturation of the endogenous $\mathrm{Ca}^{2+}$ buffer therefore might produce the apparent facilitation of $\Delta\left[\mathrm{Ca}^{2+}\right]_{\mathrm{tr}}$. The linear relationship between $I_{\mathrm{Ca}}$ and $\left[\mathrm{Ca}^{2+}\right]_{\mathrm{i}}$ observed in these nerve terminals using Fura-2 (Stuenkel, 1994, his Fig. 2) suggests that $\mathrm{Ca}^{2+}$ influx does not saturate the endogenous buffer under these conditions. Furthermore, numerical calculations, using the above-mentioned one-compartment model (Regehr et al., 1994; Tank et al., 1995), indicate that buffer saturation results in a sigmoidal shape for $\left[\mathrm{Ca}^{2+}\right]_{\text {res }}$, which was not observed in our experiments. Given the slow time course of $\mathrm{Ca}^{2+}$ extrusion and/or uptake (see below), these factors are also unlikely to affect the time course or amplitudes of $\Delta\left[\mathrm{Ca}^{2+}\right]_{\mathrm{tr}}$.

This leaves changes in $\mathrm{Ca}^{2+}$ influx and, potentially, $\mathrm{Ca}^{2+}$ mobilization as the relevant sources for the observed changes in $\Delta\left[\mathrm{Ca}^{2+}\right]_{\text {tr }}$ amplitudes. The role of $\mathrm{Ca}^{2+}$ influx in excitationsecretion coupling is well established (Douglas, 1963; Douglas and Poisner, 1964). Release of $\mathrm{Ca}^{2+}$ from intracellular stores is tightly coupled to secretion in many other secretory cells (Tse and Tse, 1999). Mobilization of $\mathrm{Ca}^{2+}$ from isolated granules themselves, for example, has been reported in pancreatic acinar (Gerasimenko et al., 1996) and chromaffin cells (Yoo and Albanesi, 1990). We have recently localized both Type 2 Ryanodine and Type $1 \mathrm{InsP}_{3} \mathrm{Ca}^{2+}$ release channels on the dense-core secretory granules in the mouse neurohypophysis (Salzberg et al., 2000b). Together with reports of high $\mathrm{Ca}^{2+}$ concentrations in these granules (Thirion et al., 1997), the possibility of $\mathrm{Ca}^{2+}$ mobilization from secretory granules (or other intraterminal stores) cannot be excluded. However, because there is as yet no direct evidence for $\mathrm{Ca}^{2+}$ release from intraterminal compartments in the neurohypophysis, we will treat the terms " $\mathrm{Ca}^{2+}$ influx" and " $\Delta\left[\mathrm{Ca}^{2+}\right]_{\mathrm{tr}}$ " as equivalent.

\section{$\mathrm{Ca}^{2+}$ extrusion or uptake}

Figure 3 shows the $\mathrm{Ca}^{2+}$ decay $\left(\Delta\left[\mathrm{Ca}^{2+}\right]_{\mathrm{dec}}\right)$ immediately after the last $\Delta\left[\mathrm{Ca}^{2+}\right]_{\text {tr }}$ rise, on a linear (Fig. $3 A$ ) and a logarithmic (Fig. $3 B$ ) time scale. As is apparent from Figure $3 A, \Delta\left[\mathrm{Ca}^{2+}\right]_{\text {dec }}$ displays a bimodal decay pattern: an initial rapid decay switches over to a very slow decay as $\left[\mathrm{Ca}^{2+}\right]_{\mathrm{i}}$ approaches resting levels. The initial rapid decay phase, in turn, contains two distinct components. Figure $3 D$ summarizes the results from double-exponential fits to this initial decay phase. The two components of the rapid decay phase display a significantly different dependence on residual calcium levels. The time constant $\tau_{\text {fast }}^{1}$ of the faster component increases from a low of $\sim 25 \mathrm{msec}$ to a maximum of $200 \mathrm{msec}$ at the highest level of residual calcium. This increase is directly reflected in Figure $3 B$ in the increasing delay before the onset of the steep decay. Any single $\mathrm{Ca}^{2+}$-sensitive $\mathrm{Ca}^{2+}$ extrusion/uptake mechanism would be expected to increase its macroscopic rate of $\mathrm{Ca}^{2+}$ extrusion with increasing $\mathrm{Ca}^{2+}$ load. The observed decrease, therefore, might result from a transition between two distinct extrusion mechanisms with separate times scales of $\tau^{1}$ fast $(\min )$ and $\tau_{\text {fast }}^{1}(\max )$, respectively.

In contrast, the time constant of the second fast component, $\tau_{\text {fast }}^{2}$, remains fairly constant at $400 \pm 50 \mathrm{msec}$. The slow decay is not well resolved in this record and, for the purpose of the doubleexponential fit shown in Figure 3, was set equal to a constant background. Quantitative measurement of the slow time constant was hampered by its sensitivity to small errors in the bleaching correction (see Materials and Methods). Nevertheless, from extended $\Delta F / F_{0}$ recordings we estimate the slow time constant to be $\tau_{\text {slow }}=15-25 \mathrm{sec}$, i.e., 50-fold slower than the initial decay. Inspection of the raw data in Figure $3 A$ reveals clearly that the relative 
contributions of the three decay components of $\left[\mathrm{Ca}^{2+}\right]_{\mathrm{i}}$ vary with the level of residual calcium. Although the amplitudes of the slow and the second fast component both saturate around $f_{\text {Stim }}=10 \mathrm{~Hz}$, the amplitude and time constant of the first fast component increase essentially monotonically with stimulation frequency.

The presence of at least three different decay components with both amplitudes and decay rates dependent on the level of residual calcium adds another level of complexity to the temporal coding of $\left[\mathrm{Ca}^{2+}\right]_{\mathrm{i}}$ transients during stimulation trains. The dominant role of the slow decay component at low frequencies, for example, provides the terminals with a "long-term" memory for their stimulation history even after brief, low-frequency stimulation. Similarly, the changing amplitudes and time constants of the fast decay components modulate the kinetics of $\Delta\left[\mathrm{Ca}^{2+}\right]_{\mathrm{i}}$ decay, particularly within the first few stimuli of a given train of action potentials. This is highlighted in Figure $3 C$, which shows a superposition of the 1st, 5th, and 15 th response in a $15 \mathrm{~Hz}$ stimulus train. Initially, $\Delta\left[\mathrm{Ca}^{2+}\right]_{\mathrm{dec}}$ between stimuli is clearly bimodal, whereas it becomes essentially single exponential as $\tau^{1}$ fast increases beyond the interstimulus period. Without definitive pharmacological data, however, any specific assignment of different $\mathrm{Ca}^{2+}$ decay components to particular extrusion/uptake mechanisms must remain tentative.

\section{Buildup and decay of residual calcium levels}

Figure $4 A$ depicts the net increase in residual calcium during stimulation by plotting the $\Delta F / F_{0}$ values at the foot of each individual $\Delta\left[\mathrm{Ca}^{2+}\right]_{\mathrm{tr}}$ within a train. The initial mismatch between $\mathrm{Ca}^{2+}$ influx during stimulation and the rate of $\mathrm{Ca}^{2+}$ extrusion between action potentials effects this net increase in $\left[\mathrm{Ca}^{2+}\right]_{\mathrm{res}}$. For any given stimulus train, $\left[\mathrm{Ca}^{2+}\right]_{\text {res }}$ increases until the $\mathrm{Ca}^{2+}$ extrusion rate begins to match the time-averaged $\mathrm{Ca}^{2+}$ influx. This behavior is in overall agreement with a one-compartment model for $\Delta\left[\mathrm{Ca}^{2+}\right]_{\mathrm{i}}$ kinetics that predicts a single-exponential rise in $\left[\mathrm{Ca}^{2+}\right]_{\text {res }}$ (Regehr et al., 1994; Tank et al., 1995). With increasing stimulation frequency, however, single-exponential fits only conform to progressively shorter segments of the rising phase of residual calcium and overestimate its plateau amplitudes (Fig. 4B, solid lines). This deviation from the model behavior is caused by the rapid depression of calcium influx during continuous stimulation in this frequency range.

Figure $4 C$ shows both the plateau amplitude $(\square)$ and rise time (ם) of $\left[\mathrm{Ca}^{2+}\right]_{\text {res }}$ versus $f_{\text {Stim }}$. Several features are noteworthy. First, the plateau amplitude does not pass through the zero- $\mathrm{Ca}^{2+}$ origin but appears to have a finite offset. This offset is caused by the presence of the slow decay component, which keeps $\left[\mathrm{Ca}^{2+}\right]_{\text {res }}$ elevated for many tens of seconds after a stimulation train is completed. The corresponding low-frequency component of $\mathrm{Ca}^{2+}$ extrusion is not resolved on the frequency scale of our experiments. Second, the saturation of $\left[\mathrm{Ca}^{2+}\right]_{\text {res }}$ at higher frequencies arises from the pronounced depression of $\Delta\left[\mathrm{Ca}^{2+}\right]_{\mathrm{tr}}$ during actionpotential stimulation. In contrast, fixed $\Delta\left[\mathrm{Ca}^{2+}\right]_{\mathrm{tr}}$ amplitudes would result in a strictly linear increase of the $\left[\mathrm{Ca}^{2+}\right]_{\text {res }}$ plateau with frequency, as observed in the crayfish neuromuscular junction [Tank et al. (1995), their Fig. 3B]. Finally, the rise time of residual calcium is a highly nonlinear function of stimulation frequency because, apparently, increasingly effective extrusion pathways are activated during the rapid $\mathrm{Ca}^{2+}$ rise at high-frequency stimulation.

\section{Frequency dependence of AVP secretion}

Figure $5 A$ summarizes the dependence of AVP release on $f_{\text {Stim }}$. The aliquots used for the EIA analysis were obtained from the same preparation, immediately after collection of the $\Delta F / F_{0}$ data for a given frequency. Although the absolute amount of AVP release varied somewhat from one assay to the other, the relative changes in AVP release with $f_{\text {Stim }}$ were readily reproducible. Even at the lowest $f_{\text {Stim }}$ of $1 \mathrm{~Hz}$, AVP secretion was elevated well above basal release. Secretion further increased to a broad peak value around $f_{\text {Stim }}=5-10 \mathrm{~Hz}$ and then declined again. At $f_{\text {Stim }}=40 \mathrm{~Hz}$, release had declined to the levels elicited with $1 \mathrm{~Hz}$ stimulation. Because we do not have a millisecond time-resolved measure of
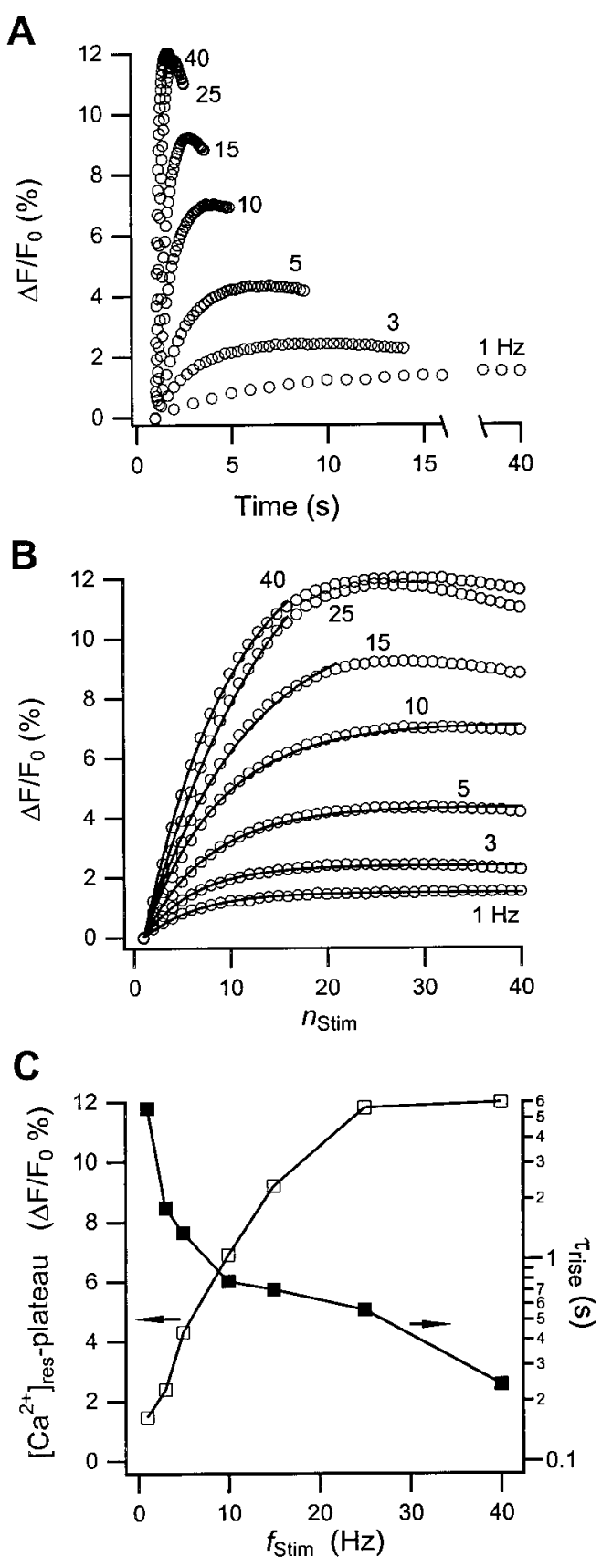

Figure 4. Changes in residual calcium during stimulation. Shown is the increase of residual calcium, $\Delta\left[\mathrm{Ca}^{2+}\right]_{\text {res }}$, in the nerve terminals of the neurohypophysis as a function of $(A)$ time and $(B)$ the stimulus number, $n_{\text {Stim }}$, during a train of 40 action potentials at the indicated $f_{\text {Stim }}$. The open circles represent the values of indicator fluorescence changes $\left(\Delta F / F_{0}\right)$ immediately before a transient $\mathrm{Ca}^{2+}$ rise. In $B$, the spacing between data points $(O)$ at different $f_{\text {Stim }}$ represents an increment in time of $\Delta \mathrm{t}=$ $\left(f_{\text {Stim }}\right)^{-1}$ sec, respectively. The solid lines represent single-exponential fits through the data. With increasing $f_{\text {Stim }}$, the range of data points conforming to this single-exponential rise rapidly decreases. $C$, Initial rise times $(\boldsymbol{\square})$ of $\Delta\left[\mathrm{Ca}^{2+}\right]_{\text {res }}$ obtained from the single-exponential fits. The open squares represent the values of the plateau amplitudes of $\Delta\left[\mathrm{Ca}^{2+}\right]_{\text {res }}$. Because the single-exponential fits did not reproduce plateau amplitudes properly, their values were instead set equal to $\Delta\left[\mathrm{Ca}^{2+}\right]_{\mathrm{res}}$ at $n_{\text {Stim }}=25$. The solid lines in $C$ are only guides to the eye.

release, we constructed a time-averaged measure for the frequency dependence of the transient and residual components of $\Delta\left[\mathrm{Ca}^{2+}\right]_{i}$ instead. Figure $5 B$ displays the cumulative amplitudes, $\Sigma\left[\mathrm{Ca}^{2+}\right]_{\mathrm{tr}}$ and $\Sigma\left[\mathrm{Ca}^{2+}\right]_{\text {res }}$, i.e., the amplitude of each component summed over the stimulus train, as a function of $f_{\text {Stim }}$. Comparison of the frequency dependence of release with that of $\Sigma\left[\mathrm{Ca}^{2+}\right]_{\operatorname{tr}}$ and 

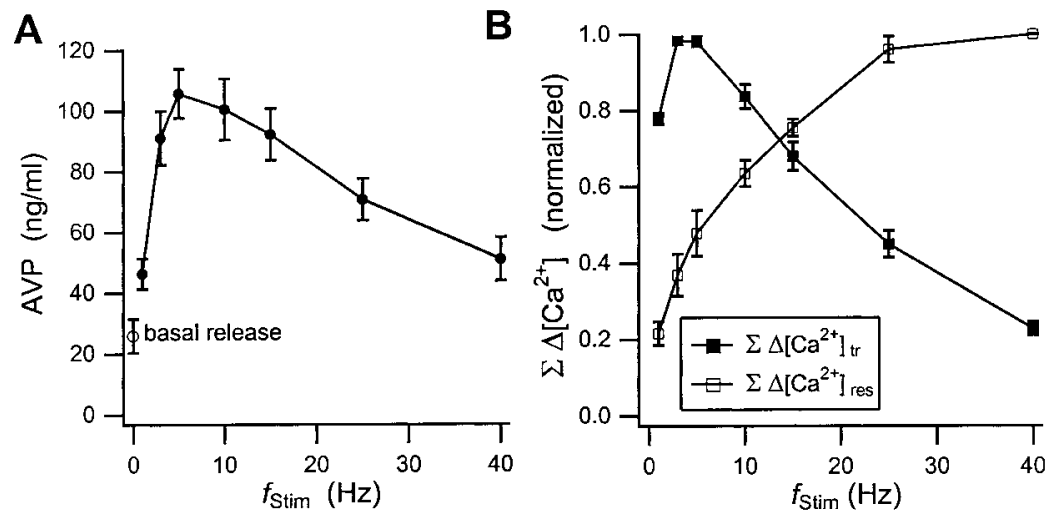

Figure 5. AVP release and cumulative $\mathrm{Ca}^{2+}$ changes. $A$, Basal release $(\bigcirc)$ and changes in AVP secretion $(\bullet)$ from the neurohypophysis in response to a train of 40 action potentials at the indicated $f_{\text {stim. }}$. The hormone levels were measured with an EIA. Aliquots from the recording chamber were collected for analysis immediately after the measurement of the stimulation-induced changes in $\left[\mathrm{Ca}^{2+}\right]_{\mathrm{i}} . B$, Relative changes in the cumulative amplitudes of the transient $\mathrm{Ca}^{2+}$ rise $\left(\Sigma \Delta\left[\mathrm{Ca}^{2+}\right]_{t+}, \mathbf{D}\right)$ during the action potential, and the cumulative residual calcium increase $\left(\Sigma \Delta\left[\mathrm{Ca}^{2+}\right]_{\mathrm{res}}, \square\right)$ throughout the stimulus train versus $f_{\text {Stim }}$. Data in $B$ are normalized to their respective peak values. Solid lines in $A$ and $B$ are guides to the eye. The error bars reflect the SDs from six different experiments.

$\Sigma\left[\mathrm{Ca}^{2+}\right]_{\text {res }}$ permits several conclusions. Clearly, $\mathrm{Ca}^{2+}$ influx during action-potential stimulation alone is sufficient to induce secretion in this preparation, as has been established for nearly 40 years (Douglas, 1963; Douglas and Poisner, 1964). More interestingly, the pattern of action-potential firing plays an active role in enhancing release through facilitation of $\mathrm{Ca}^{2+}$ rises at low-stimulation frequencies. This is particularly apparent during the steep increase in AVP release from 1 to $5 \mathrm{~Hz}$, which correlates with the pronounced $\Delta\left[\mathrm{Ca}^{2+}\right]_{\operatorname{tr}}$ facilitation in this frequency range. On the other hand, the peak in AVP secretion does not coincide with the peak in $\Sigma\left[\mathrm{Ca}^{2+}\right]_{\mathrm{tr}}$. Furthermore, for $f_{\text {Stim }}$ beyond $15 \mathrm{~Hz}, \Sigma\left[\mathrm{Ca}^{2+}\right]_{\mathrm{tr}}$ drops well below its $1 \mathrm{~Hz}$ level, whereas release remains at or above the $1 \mathrm{~Hz}$ level out to $f_{\text {Stim }}=40 \mathrm{~Hz}$. These results indicate how the increase in $\left[\mathrm{Ca}^{2+}\right]_{\text {res }}$ can sustain elevated release levels despite a substantial reduction in $\mathrm{Ca}^{2+}$ influx.

\section{Effect of burst stimulation on $\Delta\left[\mathrm{Ca}^{2+}\right]_{i}$ and AVP secretion}

We used a burst-stimulation protocol to explore the facilitation, depression, and recovery behavior of $\Delta\left[\mathrm{Ca}^{2+}\right]_{\mathrm{tr}}$. This protocol has the additional virtue that short stimulation bursts will reduce the net amplitude of $\left[\mathrm{Ca}^{2+}\right]_{\text {res }}$ and permit us to examine its relevance to AVP release. The preparation was stimulated with a continuous train of 40 stimuli at $5 \mathrm{~Hz}$ (reference) or shorter bursts of $4 \times 10$, $8 \times 5$, or $20 \times 2$ stimuli, each at an intraburst frequency of $5 \mathrm{~Hz}$, and separated by interburst intervals of 4, 2 or $1 \mathrm{sec}$, respectively. We chose $5 \mathrm{~Hz}$ as intraburst frequency because it was close to the optimal frequency for AVP release and displayed strong $\Delta\left[\mathrm{Ca}^{2+}\right]_{\mathrm{tr}}$ facilitation during continuous stimulation. Figure $6 A-C$ shows the $\Delta\left[\mathrm{Ca}^{2+}\right]_{\mathrm{tr}}$ amplitudes for these four stimulation protocols. From these traces it is apparent that facilitation and depression of $\Delta\left[\mathrm{Ca}^{2+}\right]_{\text {tr }}$ operate as separate mechanisms. For example, each one of the 10 stimuli bursts in Figure $6 A$ facilitates to a comparable degree, indicating that facilitation does not persist beyond the $4 \mathrm{sec}$ burst separation. For the shorter burst separations of $2 \mathrm{sec}$ (Fig. $6 B$, five stimuli/burst) and $1 \mathrm{sec}$ (Fig. $6 C$, two stimuli/burst), facilitation sometimes followed the reference curve for one or two bursts before falling off in subsequent bursts (data not shown). At the same time, the amplitude of the first $\mathrm{Ca}^{2+}$ transient in each subsequent burst follows the pattern of depression established by the reference record. Clearly, long-term depression persists beyond the burst separation but does not interfere with the facilitation within each burst. $\Sigma\left[\mathrm{Ca}^{2+}\right]_{\mathrm{tr}}$ amplitudes for burst stimulation drop by $\sim 10-20 \%$ from their value during continuous stimulation (Fig. $6 D$, white bars). AVP release, however, is reduced by nearly $40 \%$ (Fig. 6D, gray bars). The substantial difference between the reduction in $\Sigma \Delta\left[\mathrm{Ca}^{2+}\right]_{\text {tr }}$ and the decline in AVP secretion using burst stimulation suggests that the reduced plateau level of $\left[\mathrm{Ca}^{2+}\right]_{\text {res }}$ is the main reason for the disproportionate deterioration in AVP secretion.

\section{DISCUSSION}

At first sight, the shape of intraterminal $\mathrm{Ca}^{2+}$ changes conforms well to the predictions of a simplified one-compartment model of
$\mathrm{Ca}^{2+}$ dynamics (Regehr et al., 1994; Tank et al., 1995), which presumes fixed $\mathrm{Ca}^{2+}$ influx and a single $\mathrm{Ca}^{2+}$ extrusion mechanism. In particular, the model clearly embodies the essential features underlying the development of a steady-state plateau of residual calcium and the increase of the plateau amplitude with stimulation frequency.

On closer inspection, however, a more complex picture emerges. Most importantly, trains of action potential at different frequencies cause dramatically different patterns of $\mathrm{Ca}^{2+}$ influx. Amplitudes of $\mathrm{Ca}^{2+}$ influx can undergo pronounced facilitation and depression in the course of a single action-potential train. Dependent not only on $f_{\text {Stim }}$ but also on $N_{\text {Stim }}$, the net $\mathrm{Ca}^{2+}$ influx can be enhanced or depressed. To our knowledge, this is the first time concurrent facilitation and depression of $\mathrm{Ca}^{2+}$ influx during action-potential stimulation has been described in the neurohypophysis. In addition, $\left[\mathrm{Ca}^{2+}\right]_{i}$ decay occurs on at least three distinct, widely separated time scales. Both of these factors conspire to alter the rise times, decay times, and plateau amplitudes of residual calcium. Thus, residual calcium itself is intricately and inextricably coupled to the shifting balance between $\mathrm{Ca}^{2+}$ influx and extrusion, and the underlying pattern of action-potential stimulation. This raises the question of the physiological origin(s) of the observed changes in $\mathrm{Ca}^{2+}$ influx and $\left[\mathrm{Ca}^{2+}\right]_{\mathrm{i}}$ decay.

\section{Changes in $\mathrm{Ca}^{2+}$ influx}

Changes in the action-potential shape have long been postulated as an important factor contributing to the frequency dependence of release (Gainer et al., 1986; Bourque, 1990; Jackson et al., 1991). Conceivably, changes in action-potential shape might be sufficient to produce both facilitation and depression of $\Delta\left[\mathrm{Ca}^{2+}\right]_{\mathrm{tr}}$. For example, broadening of the action potential (Gainer et al., 1986; Bourque, 1990) might enhance the influx of $\mathrm{Ca}^{2+}$ early in a train, whereas the decrease in action-potential amplitude (Salzberg et al., 1985; Bourque, 1990) would result in depression. On the other hand, the rapid onset of facilitation of $\mathrm{Ca}^{2+}$ influx does not agree well with the gradual development of action-potential broadening [Bourque (1990), their Fig. 9].

Inactivation of $\mathrm{Ca}^{2+}$ channels might be another factor altering $\mathrm{Ca}^{2+}$ influx. Measurements on slices of the rat neurohypophysis demonstrated pronounced voltage-dependent $\mathrm{Ca}^{2+}$ channel inactivation, with recovery rates from this inhibition noticeably reduced by intraterminal $\mathrm{Ca}^{2+}$ (Branchaw et al., 1997). Hence, during low-frequency stimulation, inactivation should be minimal because the separation between action potentials is long and $\left[\mathrm{Ca}^{2+}\right]_{\text {res }}$ rises are slow and small in amplitude. As $f_{\text {Stim }}$ increases, depression sets in earlier and proceeds at a faster rate because action-potential separation decreases and $\left[\mathrm{Ca}^{2+}\right]_{\text {res }}$ rises with increasing rapidity to higher levels. This behavior agrees well with our observations (Fig. 2 ). High-frequency depression of $\mathrm{Ca}^{2+}$ influx could also result from the buildup of extracellular potassium (Frankenhaeuser and Hodgkin, 1956) during repetitive stimulation (Salzberg et al., 1985; Leng and Shibuki, 1987). Indeed, its contribution to $\mathrm{Ca}^{2+}$ influx depression might be exaggerated under our stimulation protocol as a result of the unphysiologically synchronous stimulation of the 


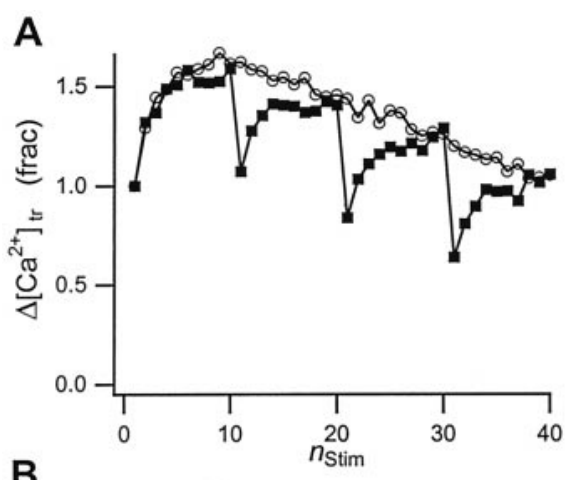

B
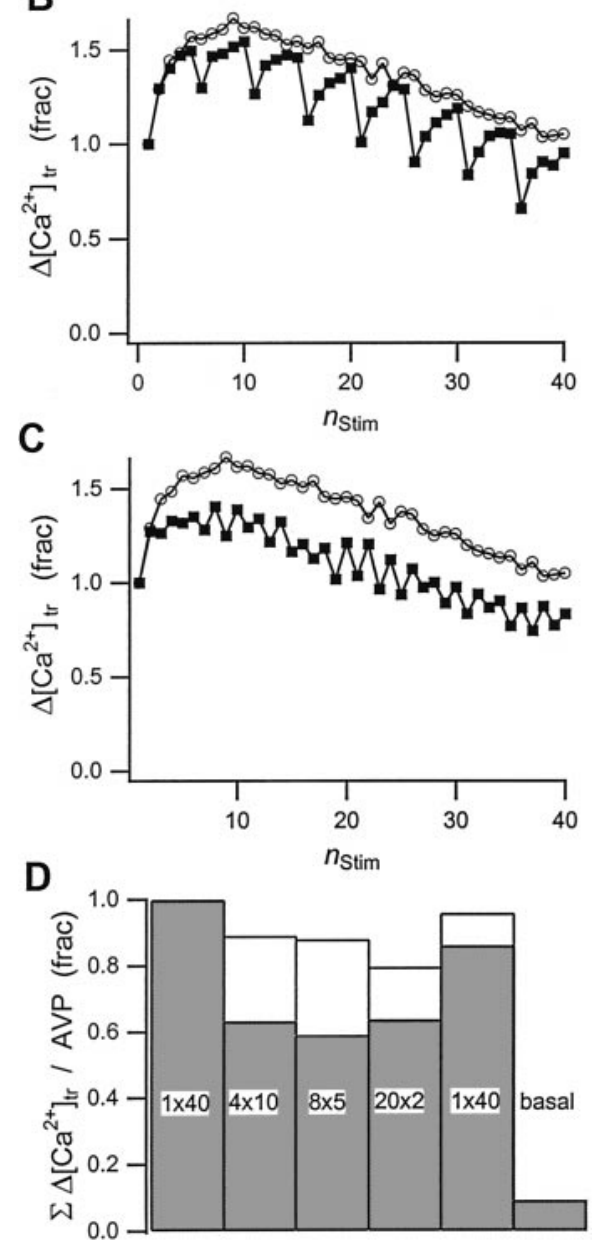

Figure 6. Effect of burst stimulation on transient $\mathrm{Ca}^{2+}$ rise and AVP release. Shown are $\Delta\left[\mathrm{Ca}^{2+}\right]_{\mathrm{tr}}$ amplitudes during stimulation of the neurohypophysis with trains of action-potential bursts at $5 \mathrm{~Hz}$. The bursts $(\square)$ consisted of either $(A) 4 \times 10,(B) 8 \times 5$, or $(C) 20 \times 2$ stimuli at $5 \mathrm{~Hz}$, separated by interburst intervals of 4,2 , and $1 \mathrm{sec}$, respectively. The reference trace $(O)$ in Figure $6 A-C$ represents the initial, continuous action-potential train of 40 stimuli at $5 \mathrm{~Hz}$. The solid lines through the data are guides to the eye. $D$, Changes in cumulative $\Delta\left[\mathrm{Ca}^{2+}\right]_{\mathrm{tr}}$ amplitudes (white bars) and AVP release (gray bars) for the data in $A-C$ with respect to the values observed with the first reference trace. Values for a second reference trace at the end of the experiments and for the basal release of AVP are shown as well. Results are normalized to the values obtained with the first reference stimulus train.

axons in the infundibular stalk. Failure of action-potential propagation (Jackson and Zhang, 1995; Obaid and Salzberg, 1996), with a reduction in the number of activated terminals, is another mechanism that could contribute to the measured depression of $\mathrm{Ca}^{2+}$ influx with increasing stimulation frequency.

Thus, there are several possible mechanisms for the depression of $\mathrm{Ca}^{2+}$ influx. The physiological origin of the observed facilitation of the $\mathrm{Ca}^{2+}$ influx is less obvious. Clearly, this is an important factor in the frequency-dependent regulation of intraterminal calcium changes and deserves additional scrutiny.

\section{$\mathrm{Ca}^{2+}$ extrusion and/or uptake}

The engagement of different $\mathrm{Ca}^{2+}$ extrusion/uptake pathways with different pump rates and $\mathrm{Ca}^{2+}$ sensitivities has to be considered an active part of the cellular control of $\mathrm{Ca}^{2+}$ dynamics during exocytosis. For example, an important transient release component of fast synaptic transmission depends critically on the decay rate of $\left[\mathrm{Ca}^{2+}\right]_{\text {res }}$ (Chen and Regehr, 1999). In the neurohypophysis, the most obvious effect of changes in $\mathrm{Ca}^{2+}$ extrusion/uptake dynamics is on the buildup and decay of residual $\mathrm{Ca}^{2+}$ levels during repetitive stimulation.

Our attempts to identify the different sources of $\mathrm{Ca}^{2+}$ extrusion/ uptake pharmacologically have been clouded by uncertainties regarding drug permeance across the plasma membrane, or by direct drug interference with excitability in the intact tissue. A comprehensive patch-clamp study on $\mathrm{Ca}^{2+}$ buffering and removal in isolated nerve terminals identified mitochondrial (ruthenium red sensitive) uptake as the dominant pathway of $\mathrm{Ca}^{2+}$ removal during repetitive depolarization, with minor contributions from $\mathrm{Ca}^{2+}$ ATPases (Stuenkel, 1994). Mitochondrial $\mathrm{Ca}^{2+}$ uptake during exocytosis has also been observed in bullfrog presynaptic peptidergic terminals (Peng, 1998). Secretory granules themselves might contribute to $\mathrm{Ca}^{2+}$ uptake in nerve terminals as well (Troadec et al., 1998). These findings would suggest that the slow extrusion in our data is associated with a plasmalemmal $\mathrm{Ca}^{2+}$ ATPase, whereas the fast component(s) reflects high-capacity, low-affinity uptake processes inside the terminals.

\section{AVP release and $\Delta\left[\mathrm{Ca}^{2+}\right]_{i}$}

Several hypotheses, most prominently action-potential broadening (Gainer et al., 1986; Bourque, 1990; Jackson et al., 1991) and buildup of residual calcium (Jackson et al., 1991; Stuenkel and Nordmann, 1993; Stuenkel, 1994), have been proposed to explain the frequency dependence of AVP release from the neurohypophysis. None of these hypotheses directly addressed the issue of why release should be optimal at any specific frequency, rather than simply increasing monotonically with frequency. The results of our $\Delta\left[\mathrm{Ca}^{2+}\right]_{\mathrm{i}}$ measurements suggest a compelling solution to this question. AVP secretion is sensitive to both the $\mathrm{Ca}^{2+}$ rise during action potentials and the amplitude of residual $\mathrm{Ca}^{2+}$ levels between action potentials. Action-potential stimulation facilitates $\mathrm{Ca}^{2+}$ rises at low $f_{\text {Stim }}$ but eventually depresses $\mathrm{Ca}^{2+}$ rises with increasing $f_{\text {Stim }}$. In contrast, residual $\mathrm{Ca}^{2+}$ increases monotonically throughout the whole frequency range. Therefore, AVP release should be optimized at some frequency beyond the maximum in $\mathrm{Ca}^{2+}$ influx, consistent with the release data in this and many previous experiments (Cazalis et al., 1985; Gainer et al., 1986; Bicknell, 1988). The data also suggest a patterning of action potentials for optimizing AVP release within any given burst: initial high-frequency stimulation to quickly raise residual $\mathrm{Ca}^{2+}$, followed by stimulation in the maximal range of release around 3-10 $\mathrm{Hz}$. This protocol precisely matches the firing pattern reported for AVP neurons in the hypothalamus (Poulain and Wakerley, 1982). Finally, the extended interburst quiescence observed in AVP neurons fits well with the extended recovery period required to overcome long-term depression of $\mathrm{Ca}^{2+}$ influx. It remains to be seen how $\Delta\left[\mathrm{Ca}^{2+}\right]_{\text {tr }}$ and $\Delta\left[\mathrm{Ca}^{2+}\right]_{\text {res }}$ interact and how they couple into various components of the secretory pathway to accomplish the frequency modulation of release.

\section{REFERENCES}

Almers W, Neher E (1985) The Ca signal from fura-2 loaded mast cells depends strongly on the method of dye loading. FEBS Lett 192:13-18.

Baylor SM, Hollingworth S (1988) Fura-2 calcium transients in frog skeletal muscle fibres. J Physiol (Lond) 403:151-192.

Bicknell RJ (1988) Optimizing release from peptide hormone secretory nerve terminals. J Exp Biol 139:51-65.

Blaustein MP, Hodgkin AL (1969) The effect of cyanide on the efflux of calcium from squid axon. J Physiol (Lond) 200:497-527. 
Bourque CW (1990) Intraterminal recordings from the rat neurohypophysis in vitro. J Physiol (Lond) 421:247-262.

Branchaw JL, Banks MI, Jackson MB (1997) $\mathrm{Ca}^{2+}$ - and voltagedependent inactivation of $\mathrm{Ca}^{2+}$ channels in nerve terminals of the neurohypophysis. J Neurosci 17:5772-5781.

Cazalis M, Dayanithi G, Nordmann JJ (1985) The role of patterned burst and interburst interval on the excitation-coupling mechanism in the isolated rat neural lobe. J Physiol (Lond) 369:45-60.

Chan DW (1987) Immunoassay: a practical guide. Orlando, FL: Academic.

Chen C, Regehr WG (1999) Contributions of residual calcium to fast synaptic transmission. J Neurosci 19:6257-6266.

Crank J (1975) The mathematics of diffusion, Ed 2. New York: Oxford UP.

Douglas WW (1963) A possible mechanism of neurosecretion-release of vasopressin by depolarization and its dependence on calcium. Nature 197:81-82.

Douglas WW, Poisner AM (1964) Stimulus-secretion coupling in a neurosecretory organ and the role of calcium in the release of vasopressin from the neurohypophysis. J Physiol (Lond) 172:1-18.

Fisher TE, Fernandez JM (1999) Pulsed laser imaging of $\mathrm{Ca}^{2+}$ influx in a neuroendocrine terminal. J Neurosci 19:7450-7457.

Frankenhaeuser B, Hodgkin AL (1956) The after-effects of impulses in the giant nerve fibres of Loligo. J Physiol (Lond) 131:341-376.

Gainer H, Wolfe Jr SA, Obaid AL, Salzberg BM (1986) Action potentials and frequency-dependent secretion in the mouse neurohypophysis. Neuroendocrinology 43:557-563.

Gerasimenko OV, Gerasimenko JV, Belan PV, Petersen OH (1996) Inositol trisphosphate and cyclic ADP-ribose-mediated release of $\mathrm{Ca}^{2+}$ from single isolated pancreatic zymogen granules. Cell 84:473-480.

Haugland RP (1996) Handbook of fluorescent probes and research chemicals, Ed 6. Eugene, OR: Molecular Probes.

Helmchen F, Borst JGG, Sakmann B (1997) Calcium dynamics associated with a single action potential in a CNS presynaptic nerve terminal. Biophys J 72:1458-1471.

Jackson MB, Zhang SJ (1995) Action potential propagation and propagation block by GABA in rat posterior pituitary nerve terminals. J Physio (Lond) 483:597-611.

Jackson MB, Konnerth A, Augustine GJ (1991) Action potential broadening and frequency-dependent facilitation of calcium signals in pituitary nerve terminals. Proc Natl Acad Sci USA 88:380-384.

Leng G, Shibuki K (1987) Extracellular potassium changes in the rat neurohypophysis during activation of the magnocellular neurosecretory system. J Physiol (Lond) 392:97-111.

Muschol M, Salzberg BM (2000) Frequency dependence of intraterminal calcium changes and vasopressin release in the neurohypophysis. Biophys J 78:260A.

Muschol M, Dasgupta BR, Salzberg BM (1999) Calcium and barium kinetics in mammalian nerve terminals during exocytosis. Biophys $\mathrm{J}$ 76:A400.

Nordmann JJ (1977) Ultrastructural morphometry of the rat neurohypophysis. J Anat 123:213-218.

Obaid AL, Salzberg BM (1996) Micromolar 4-aminopyridine enhances invasion of a vertebrate neurosecretory terminal arborization: optical recording of action potential propagation using an ultrafast photodiodeMOSFET camera and a photodiode array. J Gen Physiol 107:353-368.
Obaid AL, Flores R, Salzberg BM (1989) Calcium channels that are required for secretion from intact nerve terminals of vertebrates are sensitive to $\omega$-conotoxin and relatively insensitive to dihydropyridines. J Gen Physiol 93:715-729.

Peng YY (1998) Effects of mitochondrion on calcium transients at intact presynaptic terminals depend on frequency of nerve firing. J Neurophysiol 80:186-195.

Poulain DA, Wakerley JB (1982) Electrophysiology of hypothalamic magnocellular neurones secreting oxytocin and vasopressin. Neuroscience 7:773-808.

Regehr WG, Delaney KR, Tank DW (1994) The role of presynaptic calcium in short-term enhancement at the hippocampal mossy fiber synapse. J Neurosci 14:523-537.

Salzberg BM, Obaid AL, Senseman DM, Gainer H (1983) Optical recording of action potentials from vertebrate nerve terminals using potentiometric probes provides evidence for sodium and calcium components. Nature 306:36-40.

Salzberg BM, Obaid AL, Gainer H (1985) Large and rapid changes in light scattering accompany secretion by nerve terminals in the mammalian neurohypophysis. J Gen Physiol 86:395-411.

Salzberg BM, Muschol M, Obaid AL (2000a) Rapid changes in light scattering associated with secretion from peptidergic nerve terminals. In: Imaging neurons. A laboratory manual (Yuste R, Lanni F, Konnerth A, eds), pp 48.1-48.8. Cold Spring Harbor, NY: Cold Spring Harbor Laboratory.

Salzberg BM, Muschol M, Kraner SD, Obaid AL (2000b) Localization of calcium release channels to secretory granules in terminals of the mouse neurohypophysis. Biophys J 78:260A.

Sattelle DB (1988) Quasielastic laser light scattering and laser doppler electrophoresis as probes of synaptic and secretory terminal function. J Exp Biol 139:233-253.

Stuenkel EL (1994) Regulation of intracellular calcium and calcium buffering properties of rat isolated neurohypophysial nerve endings. J Physiol (Lond) 481:251-271.

Stuenkel EL, Nordmann JJ (1993) Intracellular calcium and vasopressin release of rat isolated neurohypophysial nerve endings. J Physiol (Lond) 468:335-355.

Tank DW, Regehr WG, Delaney KR (1995) A quantitative analysis of presynaptic calcium dynamics that contribute to short-term enhancement. J Neurosci 15:7940-7952.

Thirion S, Troadec JD, Pagnotta S, Andrews SB, Leapman RD, Nicaise G (1997) Calcium in secretory vesicles of neurohypophysial nerve endings: quantitative comparison by X-ray microanalysis of cryosectioned and freeze-substituted specimens. J Microsc 186:28-34.

Troadec JD, Thirion S, Laugier JP, Nicaise G (1998) Calcium-induced calcium increase in secretory vesicles of permeabilized rat neurophyophysial nerve terminals. Biol Cell 90:339-347.

Tse FW, Tse A (1999) Regulation of exocytosis via release of $\mathrm{Ca}^{2+}$ from intracellular stores. BioEssays 21:861-865.

Wang G, Dayanithi G, Kim S, Horn D, Nadasdi L, Kristipati R, Ramachandran J, Stuenkel EL, Nordmann JJ, Newcomb R, Lemos JR (1997) Role of Q-type channels in vasopressin secretion from neurohypophysial terminals of the rat. J Physiol (Lond) 502:351-362.

Yoo SH, Albanesi JP (1990) Inositol 1,4,5-trisphosphate-triggered $\mathrm{Ca}^{2+}$ release from bovine adrenal medullary secretory vesicles. J Biol Chem 265:13446-13448. 